\title{
PROPOSAL FOR A RACE-TRACK MICROTRON WITH HIGH PEAK CURRENT *
}

\author{
G.J. ERNST, E.H. HASELHOFF and W.J. WITTEMAN \\ Unwersity of Twente, Department of Applied Physics, 7500 AE Enschede, The Netherlands
}

\section{J.I.M. BOTMAN, W. VAN GENDEREN, H.L. HAGEDOORN, J.A. VAN DER HEIDE and W.J.G.M. KLEEVEN}

Eindhoven Unversity of Technology, Cyclotron Laboratory, $5600 \mathrm{MB}$ Eindhoven, The Netherlands

\begin{abstract}
In order to obtain high gain in a free electron laser a high-quality electron beam with high peak current is required. It is well-known that a microtron is able to produce a high-quality beam having low emittance and small energy spread (1\%). Because a curcular microtron has a limited high-current capability a race-track design is adopted for providing flexibility, better beam quality and of course higher peak current in the microbunch. Space charge problems may be severe in a microtron. It can be shown that bunching on certain specific subharmonic frequencies will lead to a strong reduction of the space charge problems. The general layout of our microtron design will be presented. The charactenstics are: energy $25 \mathrm{MeV}$, micropulse $10^{\circ}$ of the rf frequency of $3 \mathrm{GHz}$. Our aim is to come beyond the present state of the art with the following characteristics: relative energy spread 0.001 , emittance $3 \mathrm{~mm}$ mrad, current in the micropulse $100 \mathrm{~A}$, macropulse length $50 \mu \mathrm{s}$ and subharmonic bunching at $1: 64$.
\end{abstract}

\section{Introduction}

Shortcomings of existing free electron lasers in the visible and near infrared region of the spectrum are the limited frequency tunability and the large bandwidth of their produced laser radiation. This is due to the small gain coefficient and the time structure of the electron pulses of those systems. Improvements can be expected if high-current, high-quality electron beams are used so that it is possible to insert optical elements - e.g. dispersive elements to provide tuning - into the cavity. Moreover it is then possible to delay the laser pulses with respect to the electron pulses or vice versa for instance by detuning the laser cavity length from its optimum value. This leads of course to a reduction in gain but also to an increased laser pulse length and therefore to an improved radiation bandwidth.

Looking at the different possibilities we think that a race-track (RT) microtron is well suited for obtaining our goal described above, because it combines the high current capability of a linac with the excellent beam properties of a circular microtron.

In this paper aspects are described of our proposed RT microtron. The same concept but with about twice the electron energy will be used as injector for the proposed electron storage ring EUTERPE [1]. The FEL will operate in the infrared e.g. for studies of isotope

* This work is supported by Ultra-Centrifuge Nederland (UCN), Almelo, The Netherlands. separation. Standard rf components at $3 \mathrm{GHz}$ will be used.

Typical electron energy and undulator wavelength are $25 \mathrm{MeV}$ and $30 \mathrm{~mm}$. For our specifications a study of space charge effects will be crucial. Strong axial focusing will be supplied as well as means to adjust the isochronism. Space charge problems will be largest at those places where the successive orbits overlap. However, a computer analysis shows that injection at specific subharmonic frequencies reduces those problems.

\section{General layout of the microtron}

The overall layout of the microtron is given in fig. 1 . A parameter list is given in table 1. The machine has two magnetic sectors separated by a distance of $0.5 \mathrm{~m}$. The space in between contains the rf cavity, the injection and extraction magnets, and a small general corrector magnet. A $5 \mathrm{MeV}$ injector precedes the microtron. The orbit separation is $3 \mathrm{~cm}$ which is enough for placing the injector and extraction magnet. Axial stability is obtained by edge focusing: a magnetic valley in the magnet sector pole pieces is provided under $45^{\circ}$ with the sector edges.

The present microtron parameters require $17 \mathrm{rf}$ wavelengths on the first turn, corresponding to an injector energy of about $5 \mathrm{MeV}$, and one wavelength increment between successive turns. For energies from 5 to $10 \mathrm{MeV}$ there is still a noticeable variation in $\beta=v / c$. Corrector coils in the pole piece valleys will be used for 


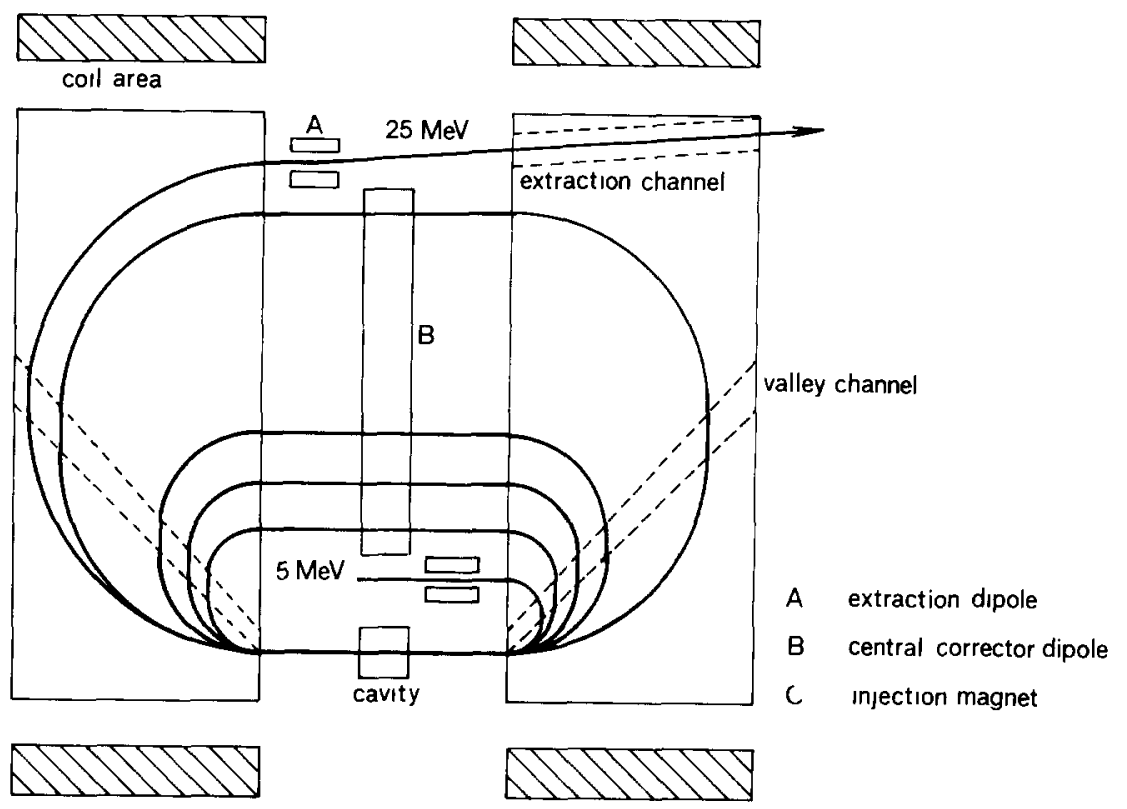

Fig. 1. Microtron layout.

turn by turn adjustment of the synchronism. More details of the microtron can be found in ref. [2].

\section{Pulse selection}

When no pulse selection is applied, for 19 turns and thus 20 accelerations, there are 20 pulses simultaneously present in the cavity and the common straight section. Space charge effects increase when pulses overlap, thus setting a limit to the maximum peak current. However, in a RT microtron, in contrast to a circular one, external injection can be used and therefore pulse selection can be applied.

The synchronization condition requires that the travel time of the electrons for an orbit increases by a whole

Table 1

Microtron parameter list

\begin{tabular}{ll}
\hline Energy range & $5-25(50) \mathrm{MeV}$ \\
Number of turns & 20 \\
Magnetic field & $0.2(0.4) \mathrm{T}$ \\
Cavity voltage & $1(2) \mathrm{MV}$ \\
Frequency & $3 \mathrm{GHz}$ \\
Peak current & $100 \mathrm{~A} /$ pulse \\
Rf power & $4 \mathrm{MW}$ in $50 \mu \mathrm{s}$ \\
Pulse selection & $1: 64$ \\
Orbit separation & $3 \mathrm{~cm}$ \\
Magnet sectors separation & $50 \mathrm{~cm}$ \\
\hline
\end{tabular}

number of rf-periods $T_{\mathrm{rf}}$ after each acceleration, or:

$T_{n+1}-T_{n}=\beta T_{\mathrm{rf}}$,

where $T_{n}$ is the travel time for the $n$th orbit.

Moreover the travel time for the first orbit, i.e. after the first acceleration, also has to equal a whole number of rf-periods:

$T_{1}=(\alpha+\beta) T_{\mathrm{rf}}$.

For the total number of rf-periods inside the microtron it follows:

$$
\begin{aligned}
N & =\sum_{n=1}^{k} \frac{T_{n}}{T_{\mathrm{rf}}}=\sum_{n=1}^{k} \alpha+n \beta=k \alpha+\beta \sum_{n=1}^{k} n \\
& =k \alpha+\frac{1}{2} k(k+1) \beta,
\end{aligned}
$$

where $k$ is the total number of orbits.

If at a certain time an electron bunch is injected into the cavity, it will enter the cavity and be accelerated for the last time after $N+1 \mathrm{rf}$ periods. Thus, if every $N+2$ rf periods an electron bunch is injected, overlapping of bunches can never occur. This, however, is unpractical because usually $N$ is large. For instance for our case with $k=19, \beta=1$ and $\alpha=15, N$ equals 475 . That 1s why we now investigate injection with a rate faster than 1 out of $N+2$. Suppose the injection rate is 1 out of $N_{1}$ and $N_{\mathrm{g}}$ rf periods are passed after injection. The bunches are present inside the microtron at $\mathrm{rf}$ "positions":

$N_{\mathrm{g}}+{ }_{l} N_{\iota}$, with $0 \leq \imath \leq \frac{N+1-N_{\mathrm{g}}}{N_{\iota}}$,

$\imath$ being an integer. 
Each position is determined by the number of rfwavelengths along the electron path, starting with position 0 at the rf-cavity for injected electrons.

The following positions coincide with the rf-cavity position:

$j \boldsymbol{\alpha}+\frac{1}{2} J(J+1) \beta$, with $0 \leq J \leq k$,

$J$ being an integer.

So it is possible to count, for every value of $N_{\mathrm{g}}$ for a fixed $N_{\iota}$ value, the number of bunches simultaneously present inside the cavity. Because those numbers are periodic with period $N_{l}$ it is obvious that a complete set of numbers is found for $N_{\mathrm{g}}$ running from 0 up to $N_{\imath}-1$.

It turns out that for certain injection rates it never happens that two electron bunches are simultaneously present inside the cavity. For our numbers of $k, \alpha$ and $\beta$ the fastest rate for which this happens is 1 out of 46 . The next rates are 1 out of $59,61,64,67$, and so on.

The values of those specific rates are fundamental microtron parameters and do not depend on the particular value of $\alpha$ and $k$. However, each specific rate has its own range of $\alpha$ and $k$ for which bunch overlap in the cavity never occurs. For instance 1 out of 46 can be used for all $k$-values smaller than 21, whereas $\alpha$-values as low as 10 can be used. And of course for smaller values of $k$ even faster injection rates than 1 out of 46 can be used.
Table 3

The number of bunches simultaneously present at the cavity position for successive accelerations $J$ of a particular bunch for three different injection rates: 1 out of $63(=\alpha), 1$ out of 64 $(=\beta)$ and 1 out of $65(=\gamma)$.

\begin{tabular}{rlll}
\hline$j$ & $\alpha$ & $\beta$ & $\gamma$ \\
\hline 0 & 3 & 1 & 1 \\
1 & 2 & 1 & 1 \\
2 & 1 & 1 & 1 \\
3 & 1 & 1 & 3 \\
4 & 2 & 1 & 1 \\
5 & 1 & 1 & 1 \\
6 & 1 & 1 & 1 \\
7 & 2 & 1 & 1 \\
8 & 3 & 1 & 2 \\
9 & 1 & 1 & 1 \\
10 & 2 & 1 & 1 \\
11 & 1 & 1 & 1 \\
12 & 1 & 1 & 1 \\
13 & 2 & 1 & 2 \\
14 & 3 & 1 & 1 \\
15 & 3 & 1 & 2 \\
16 & 1 & 1 & 3 \\
17 & 3 & 1 & 1 \\
18 & 3 & 1 & 3 \\
19 & 2 & 1 & 2 \\
\hline
\end{tabular}

For our microtron 1 out of 64 will be chosen because bunching at this subharmonic frequency is easy and because the optical cavity length of the FEL will be

Table 2

The number of bunches simultaneously present inside the cavity as a function of the position $N_{\mathrm{g}}$ of the injected bunch for three different mjection rates: 1 out of $63(=\alpha), 1$ out of $64(=\beta)$ and 1 out of $65(=\gamma)$.

\begin{tabular}{|c|c|c|c|c|c|c|c|c|c|c|c|}
\hline$\overline{N_{\mathrm{g}}}$ & $\alpha$ & $\beta$ & $\gamma$ & $N_{\mathrm{g}}$ & $\alpha$ & $\beta$ & $\gamma$ & $N_{\mathrm{g}}$ & $\alpha$ & $\beta$ & $\gamma$ \\
\hline 0 & 3 & 1 & 1 & 22 & 0 & 0 & 0 & 44 & 0 & 0 & 0 \\
\hline 1 & 0 & 0 & 0 & 23 & 0 & 0 & 0 & 45 & 0 & 0 & 0 \\
\hline 2 & 0 & 1 & 0 & 24 & 0 & 1 & 0 & 46 & 0 & 0 & 1 \\
\hline 3 & 0 & 0 & 1 & 25 & 0 & 1 & 1 & 47 & 0 & 1 & 0 \\
\hline 4 & 0 & 0 & 0 & 26 & 0 & 1 & 2 & 48 & 1 & 0 & 0 \\
\hline 5 & 0 & 1 & 1 & 27 & 1 & 1 & 0 & 49 & 0 & 0 & 0 \\
\hline 6 & 1 & 1 & 0 & 28 & 0 & 1 & 0 & 50 & 0 & 0 & 1 \\
\hline 7 & 2 & 0 & 0 & 29 & 0 & 0 & 0 & 51 & 1 & 1 & 3 \\
\hline 8 & 0 & 0 & 0 & 30 & 3 & 1 & 0 & 52 & 0 & 1 & 0 \\
\hline 9 & 0 & 0 & 0 & 31 & 0 & 0 & 0 & 53 & 0 & 0 & 0 \\
\hline 10 & 0 & 0 & 1 & 32 & 0 & 0 & 0 & 54 & 1 & 0 & 0 \\
\hline 11 & 0 & 0 & 0 & 33 & 1 & 1 & 1 & 55 & 0 & 0 & 1 \\
\hline 12 & 0 & 0 & 0 & 34 & 2 & 0 & 0 & 56 & 0 & 1 & 0 \\
\hline 13 & 0 & 1 & 0 & 35 & 0 & 0 & 0 & 57 & 0 & 1 & 0 \\
\hline 14 & 0 & 0 & 0 & 36 & 0 & 0 & 1 & 58 & 0 & 0 & 0 \\
\hline 15 & 0 & 0 & 0 & 37 & 0 & 0 & 0 & 59 & 0 & 1 & 0 \\
\hline 16 & 2 & 1 & 1 & 38 & 0 & 0 & 0 & 60 & 0 & 0 & 0 \\
\hline 17 & 0 & 0 & 0 & 39 & 0 & 1 & 0 & 61 & 1 & 0 & 0 \\
\hline 18 & 0 & 0 & 1 & 40 & 0 & 0 & 0 & 62 & 0 & 0 & 0 \\
\hline 19 & 0 & 0 & 0 & 41 & 0 & 0 & 0 & 63 & & 0 & 1 \\
\hline 20 & 0 & 0 & 2 & 42 & 1 & 0 & 0 & 64 & & & 0 \\
\hline 21 & 0 & 0 & 0 & 43 & 0 & 0 & 0 & & & & \\
\hline
\end{tabular}


about $3.2 \mathrm{~m}$, which is a convenient length. Table 2 shows the number of bunches simultaneously present inside the cavity as a function of $N_{\mathrm{g}}$ for $N_{\mathrm{g}}$ running from 0 up to $N_{1}-1$ for three different values of $N_{1}$, viz. 63,64 and 65 . It is clear from the table that 1 out of 64 is the best injection rate out of the three.

It is also very instructive to follow one particular bunch and to see how many pulses are simultaneously present inside the cavity at each acceleration. Successive accelerations take place if the bunch is at the rf positions given by eq. (5), with $j$ running from 0 to $k, j$ being a whole number. Then bunches are present at rf-positions:

$i N_{l}$, with $0 \leq \imath \leq \frac{N+1}{N_{\imath}}$,

$i$ being an integer.

Now it is possible for ever $j$ value to count the total number of bunches present at the cavity position. Results are shown in table 3 for $N_{1}=63,64$ and 65 .

\section{The injector}

The main characteristics of the injector are determined by the requirements of the main accelerator. Only electrons with energy and time lying within the stable energy-phase area are accepted by the microtron. For an $\mathrm{rf}$ frequency of $3 \mathrm{GHz}$ and an acceleration voltage of about $1 \mathrm{MeV}$ the maximum accepted pulse width and absolute energy spread are $\sim 15$ ps and $\sim 25$ $\mathrm{kV}$ respectively.

The design goal of the accelerator is a peak current of $100 \mathrm{~A}$ or a total charge per bunch of $1.5 \mathrm{nC}$. The FEL will operate at a wavelength of about $10 \mu \mathrm{m}$, so the emittance must be less than a few times $10^{-6} \mathrm{~m}$ rad at $25 \mathrm{MeV}$, which means a normalized emittance $\epsilon_{\mathrm{n}} \sim$ $10^{-4} \mathrm{~m} \mathrm{rad}$.
Because the emittance requirement is not extremely high, a conventional injector will be built. The scheme is shown in fig. 2. The electron source will be a gated triode delivering $5 \mathrm{~ns}, 10 \mathrm{~A}$ pulses, having a repetition rate of $1 / 64$ of $3 \mathrm{GHz}$ being $46.875 \mathrm{MHz}$, phase-locked with respect to the $3 \mathrm{GHz}$ main frequency.

A two-stage buncher will be used: one at the 64th subharmonic of $3 \mathrm{GHz}$ or $46.875 \mathrm{MHz}$ and the other one at the 16th subharmonic or $187.5 \mathrm{MHz}$, both phase-locked at the main frequency. Each stage will compress about a factor of 4 . The result will be a pulse with a width of about 300 ps and a peak current of about $100 \mathrm{~A}$. If the proposed compression factor cannot be reached because of space charge effects, a $3 \mathrm{GHz}$ buncher will be added.

A very rough estimate of the drift length can be made by following a small signal analysis.

For a sinusoidal bunching voltage, the derivative at the equilibrium position is given by

$\left(\frac{\mathrm{d} V}{\mathrm{~d} t}\right)_{\mathrm{eq}}=\omega_{\mathrm{b}} V_{0}$

where $\omega_{\mathrm{b}}$ is the bunching frequency and $V_{0}$ the peak voltage.

The voltage modulation causes an energy modulation and thus a velocity modulation. After a time span $\Delta t$, the voltage is given by $\Delta V=\omega_{\mathrm{b}} V_{0} \Delta t$, whereas the electrons, having velocity $v$, travelled a distance $\Delta s=v \Delta t$. At that position the energy of the electrons is changed by

$\Delta \gamma=\frac{e \omega_{b} V_{0}}{m_{0} c^{2}} \Delta t$

where $\gamma$ is the ratio between the relativistic electron energy and its rest mass energy.

The time needed by those electrons to reach the unmodulated ones is given by $\Delta s / \Delta v$. So the drift

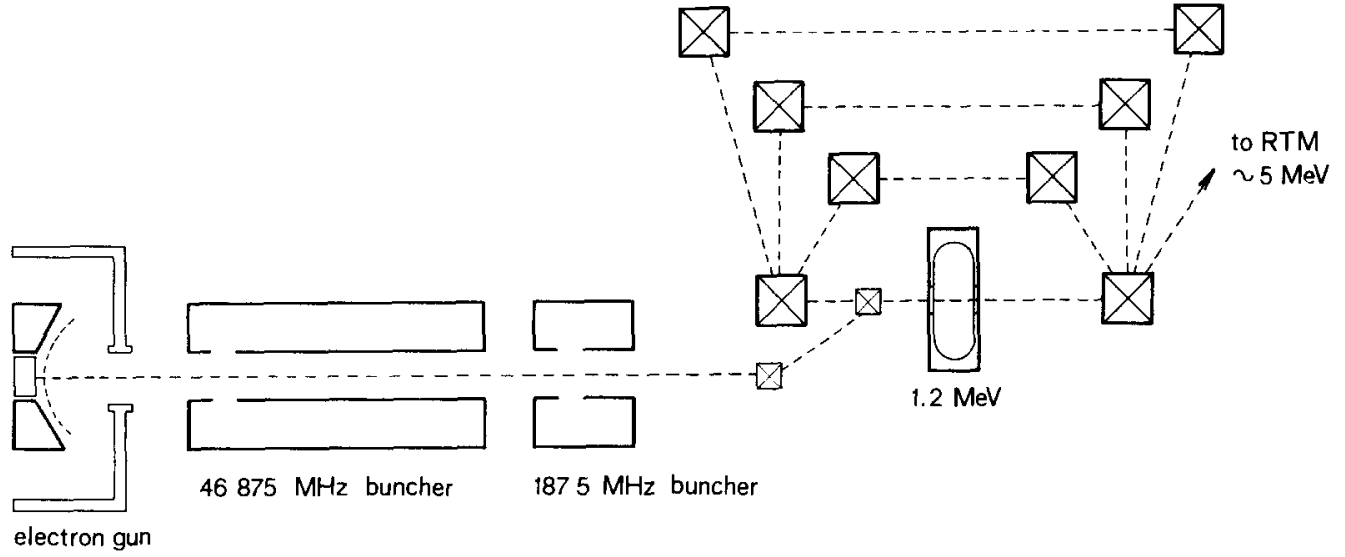

Fig. 2. Schematic layout of the preaccelerator. 
length is given by

$l_{\mathrm{d}}=v \frac{\Delta s}{\Delta v} \approx v^{2} \frac{\mathrm{d} t}{\mathrm{~d} v}=v^{2} \frac{\mathrm{d} t}{\mathrm{~d} \gamma} \frac{\mathrm{d} \gamma}{\mathrm{d} v}=c\left(\gamma^{2}-1\right)^{3 / 2} \frac{m_{0} c^{2}}{\omega_{\mathrm{b}} e V_{0}}$,

where the relation $v=c\left(1-\gamma^{-2}\right)^{1 / 2}$ has been used.

For an electron energy of $100 \mathrm{kV}$ and a modulation voltage of $30 \mathrm{kV}$ the drift length is $5 \mathrm{~m}$ for a buncher frequency of $46.875 \mathrm{MHz}$. The pulse will be injected into a circular preaccelerator. It will select the central part of the 300 ps pulse, where the energy spread is lowest, because of its stable phase area and automatically limits the energy spread to about $25 \mathrm{kV}$ as required by the RT microtron. 4 or 5 turns are necessary to obtain $5 \mathrm{MeV}$ electron energy.

\section{Rf power requirements}

The average current in our RT microtron will be limited because of the low pulse rate. For a peak current of $100 \mathrm{~A}$, a pulse rate of $46.875 \mathrm{MHz}$ and a micropulse length of $15 \mathrm{ps}$, the average current in the macropulse will be $-75 \mathrm{~mA}$. The total acceleration voltage is $25 \mathrm{MeV}$, so the average beam power is about $2 \mathrm{MW}$. We will use a Thomson tube which can deliver 4 MW of $3 \mathrm{GHz}$ power with a maximum pulse length of $100 \mu \mathrm{s}$. A repetition rate of $10 \mathrm{~Hz}$ will be used.

\section{References}

[1] J.I.M. Botman and H.L. Hagedoorn, Proc. 1987 IEEE Part. Acc. Conf., p. 488.

[2] J.I.M. Botman, W. van Genderen, H.L. Hagedorn, J.A. van der Heide, W.J.C.M. Kleeven, G.J. Ernst and W.J. Witteman, Proc. 1988 Europ. Part. Acc. Conf. 